Proceedings of the 2nd Euro-Asian Pulsed Power Conference, Vilnius, Lithuania, September 22-26, 2008

\title{
Optical Investigation of Plasma Formation Process by Interaction of Intense Electron Beams with Metallic Targets
}

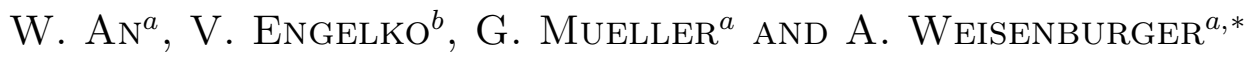 \\ ${ }^{a}$ Forschungszentrum Karlsruhe GmbH, IHM \\ P.O. Box 3640, 76021 Karlsruhe, Germany \\ ${ }^{b}$ Efremov Institute of Electrophysical Apparatus, 196641, St. Petersburg, Russia
}

\begin{abstract}
Electron beams with energies $\geq 100 \mathrm{keV}$ offer attractive possibilities to modify metallic surfaces. Numerous experiments with the electron accelerators GESA I and II showed excellent results regarding the improvement of mechanical properties and the enhancement of the corrosion resistance of the treated materials. The next step leads to further technological adaptation of the process that especially was focused on the adaptation of the electron beam to the surface geometry. For the treatment of the outer surface of tubes the electron beam facility GESA IV with a radial converged electron beam was designed. In first experiments unexpected electrical short-circuiting after different time periods occurred. Two major reasons could be identified: excessive plasma formation at the cathode and target plasma formation. The latter can be controlled by vacuum conditions and surface cleaning of the target, the first by controlling the duration of plasma formation at the cathode.
\end{abstract}

PACS numbers: 07.68.+m, 29.25.Bx, 41.75.Ak

\section{Introduction}

Electron beams with energies $\geq 100 \mathrm{keV}$ offer attractive possibilities to modify metallic surfaces. Numerous experiments with the electron accelerator GESA I and II $[1,2]$ show excellent results regarding the improvement of the mechanical property and the enhancement of the corrosion resistance of the treated materials [3-6]. Especially surface alloying of $\mathrm{Al}$ into steels to increase the steel compatibility with liquid lead alloys was successfully demonstrated [6]. One application of this surface alloyed steels is their use as fuel cladding materials in nuclear reactors [6]. The next step leads to further technological adaptation of the process that especially was focused on the adaptation of the electron beam to the surface geometry. For the treatment of the outer surface of tubes the electron beam facility GESA IV with a radial converged electron beam was designed. First experiments with the new facility showed unexpected short-circuiting. This reduces the energy transferred to the target to values not sufficient for the envisaged process. Therefore, detailed investigation of the processes applying high speed camera diagnostics was performed. The results and possible measures to overcome the problems will be addressed in this paper.

\section{GESA IV facility and diagnostics}

The GESA IV (Fig. 1b) has as a specific peculiarity a radial converging e-accelerator for treatment of outer

* corresponding author; e-mail: alfons.weisenburger@ihm.fzk.de

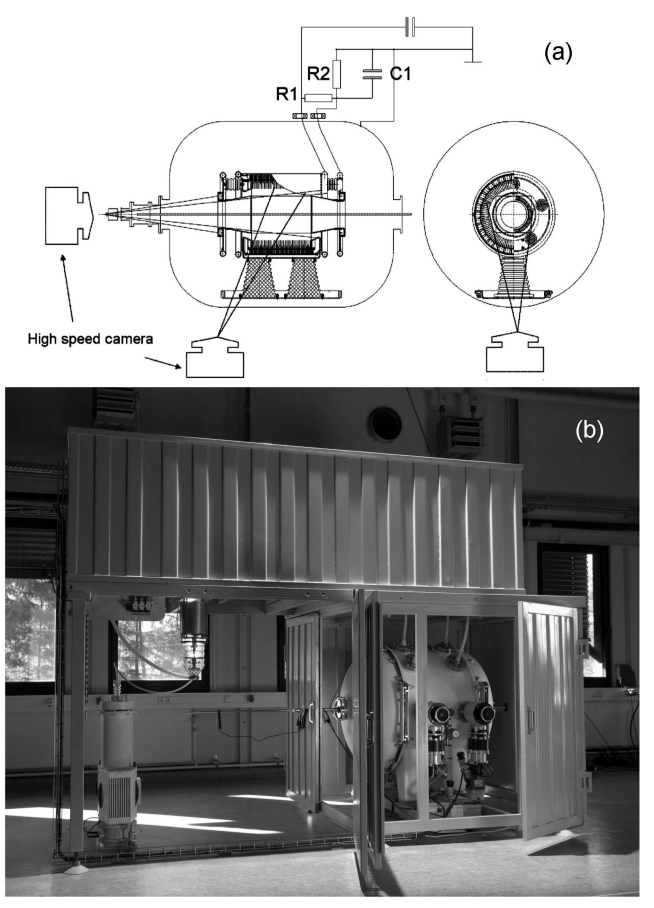

Fig. 1. (a) Scheme of GESA IV facility, (b) photo of GESA IV.

tube surfaces. Like the GESA I and II facilities $[1,2]$ the electron beam is generated by a multipoint explosive emission cathode operated in triode scheme (Fig. 1a).

The radius of the cathode made of multi carbon fibres is $140 \mathrm{~mm}$, the radius of the grid made of axial stretched wires with a transparency of $92 \%$ is $100 \mathrm{~mm}$ and the 
diameter of the target that acts here as anode is defined by the application to $8.6 \mathrm{~mm}$.

The plasma formation is studied using a high speed framing and streak camera (DiCam Pro by PCO Imaging). In first experiments the cathode area creates only a half cylinder, which allows an easier studying of the cathode and anode processes. These were observed from the unequipped lower side. Later experiments use the fully equipped cathode and the plasma formation is observed along the axis. In all experiments current and voltage of cathode, grid and target were measured.

The pressure in the working chamber was varied between $2.5 \times 10^{-5}$ and $3 \times 10^{-6}$ mbar and the grid cathode voltage ration was changed varying the resistor $\mathrm{R} 1, \mathrm{R} 2$ and the capacitor $\mathrm{C} 1$ (Fig. 1a). The values of R1, R2 and $\mathrm{C} 1$ chosen for most experiments are: $\mathrm{R} 1=34 \Omega$, $\mathrm{R} 2=564 \Omega$ and $\mathrm{C} 1=50 \mathrm{nF}$ or $100 \mathrm{nF}$.

\section{Experimental results}

Current, voltage and framing pictures of cathode and anode are monitored for all pulses to investigate the early short-circuiting of the pulses. All experimental data can be subdivided into two types.

Type 1 is characterized by a rising current right after the beginning of the pulse (Fig. 2) accompanied by excessive plasma formation at the cathode (see the streak photograph) and by relatively early short-circuiting (pulse length $<20 \mu \mathrm{s})$.

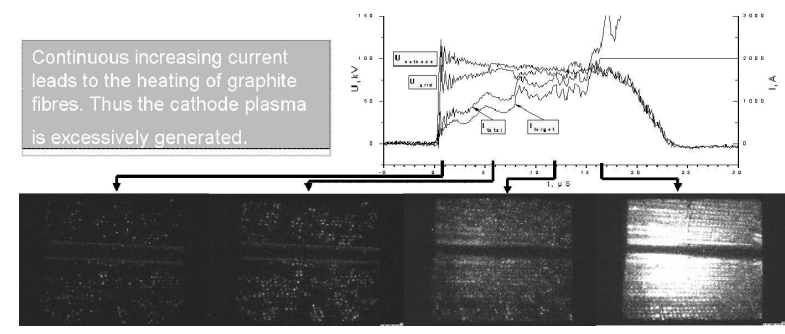

Fig. 2. Streak photograph and measured electrical signals of type 1 pulse with excessive plasma formation.

The large gap between grid voltage and cathode voltage favours highly active cathode plasma formation resulting in a disturbed balance between plasma pressure and the electrostatic pressure. The plasma front starts to move in the direction of the grid. This results in a further increase of current and finally to an impedance collapse.

Type 2 is characterized by a relatively flat and gently inclined current leading to longer pulses. Here the short-circuiting is a result of inhomogeneities of the current density at the target that leads to local increased gas desorption, ionisation and plasma formation (Fig. 3a). The geometry of the GESA IV favours a third reason for uncontrolled electrical breakdown; short-circuiting between the vacuum chamber and the grid by plasma formation at the chamber wall due to electrons leaving the cathodeanode space along the target axis (Fig. 3b). The upper framing picture is taken after about $15 \mu$ s and shows only little plasma. The lower one represents the time after the short circuiting $(\approx 21 \mu \mathrm{s})$ and clearly shows the plasma formed between the grid and the inner wall. Note that the grid current changes polarity before breakdown.

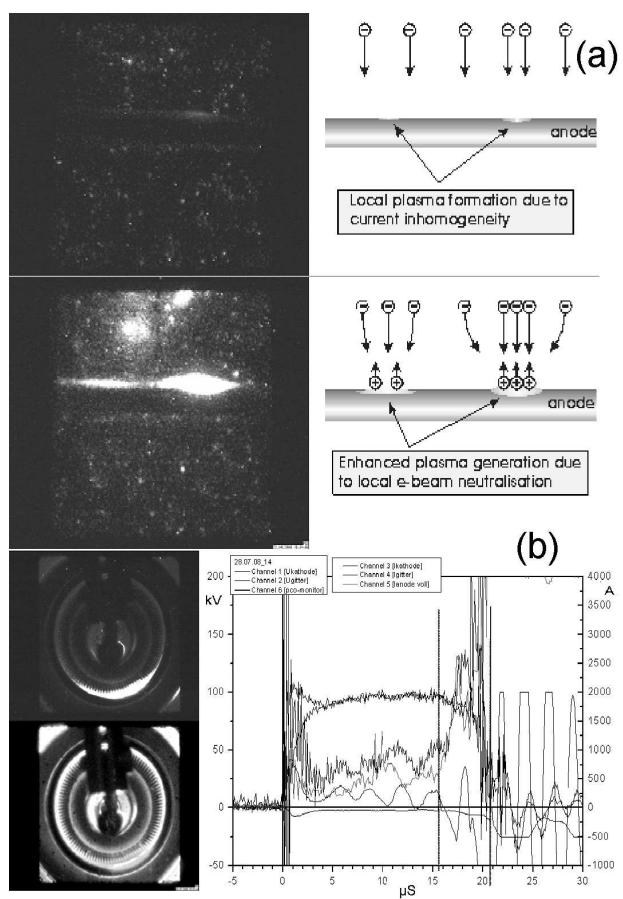

Fig. 3. (a) Type 2 pulse with plasma formation at the anode; (b) framing photograph and measured electrical signals of grid inner wall breakdown; lines indicate trigger time for framing picture (above $\approx 15 \mu \mathrm{s}$, below $\approx 21 \mu \mathrm{s})$.

\section{Measures against short-circuiting}

Changes in the Marx generator and using different vacuum conditions were investigated to overcome the first two types of pulse shortening. The capacitor $\mathrm{C} 1$ was varied form $50 \mathrm{nF}$ to $100 \mathrm{nF}$ and the resistors $\mathrm{R} 1$ and R2 were selected to the values given after numerous tests and simulations using PsPice. The simulation with $\mathrm{C} 1=50 \mathrm{nF}$ matches almost perfectly the measured values in the experiment (Fig. 5).

By changing the capacitor 1 from 50 to $100 \mathrm{nF}$ the time until the grid potential reaches the cathode potential $(\Delta U=0)$ can be varied (Fig. 4$)$.

The shorter this interval the smaller is the plasma formation and therefore the e-beam current hitting the target is reduced. Control of excessive plasma formation was achieved by reduced plasma formation due to changed electrical setup.

The control of anode or target plasma formation was investigated by changing the vacuum conditions and ap- 


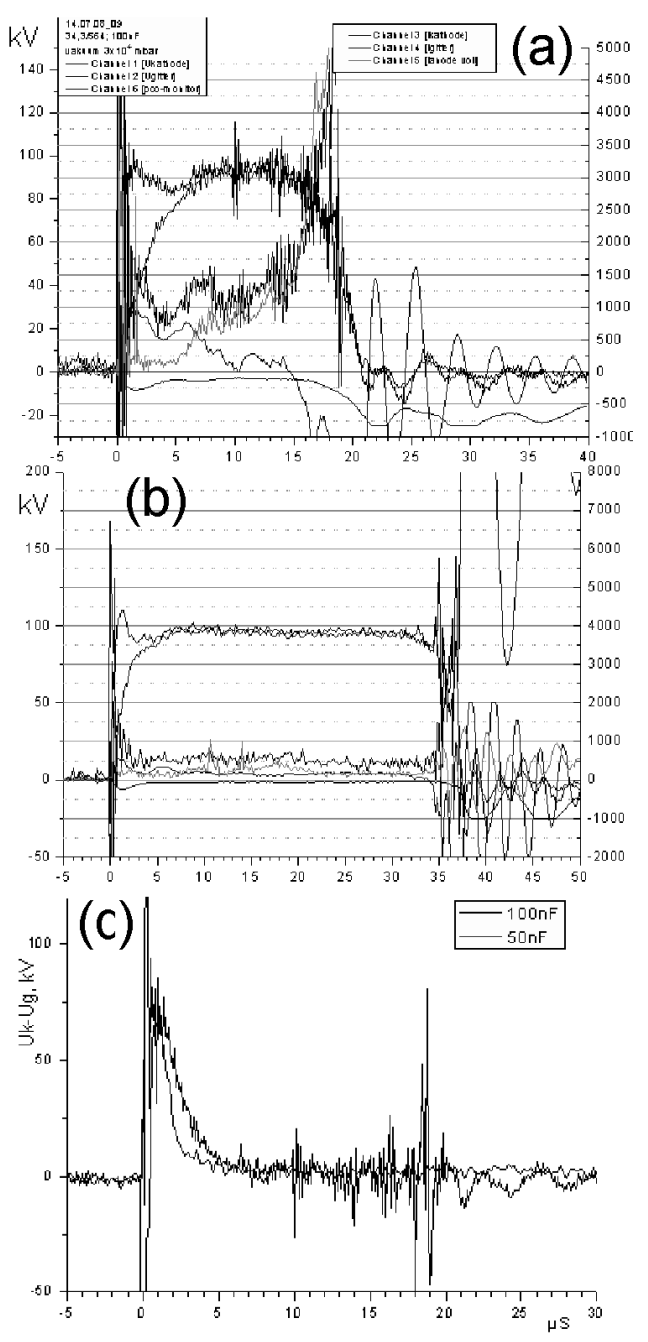

Fig. 4. Measured electrical signals: (a) $\mathrm{C} 1=50 \mathrm{nF}$, (b) $\mathrm{C} 2=100 \mathrm{nF}$, (c) $\Delta U$.

plying several pre-pulses in short time distance. The reduction of pressure from $2 \times 10^{-5}$ mbar to $3 \times 10^{-6}$ mbar leads to a significant increase in pulse duration from 25 to $60 \mu \mathrm{s}$. Formation of target plasma was drastically reduced in the low pressure regime. However, the surface state of the target (anode) is important to reach sufficiently long pulse durations. Two to three pre-pulses in a distance of about $30 \mathrm{~s}$ each were necessary to clean up the surface sufficiently.

\section{Summary and conclusions}

The excessive generation of cathode plasma can be controlled by controlling the grid potential. This results in lower beam currents and therefore in more stable pulses.

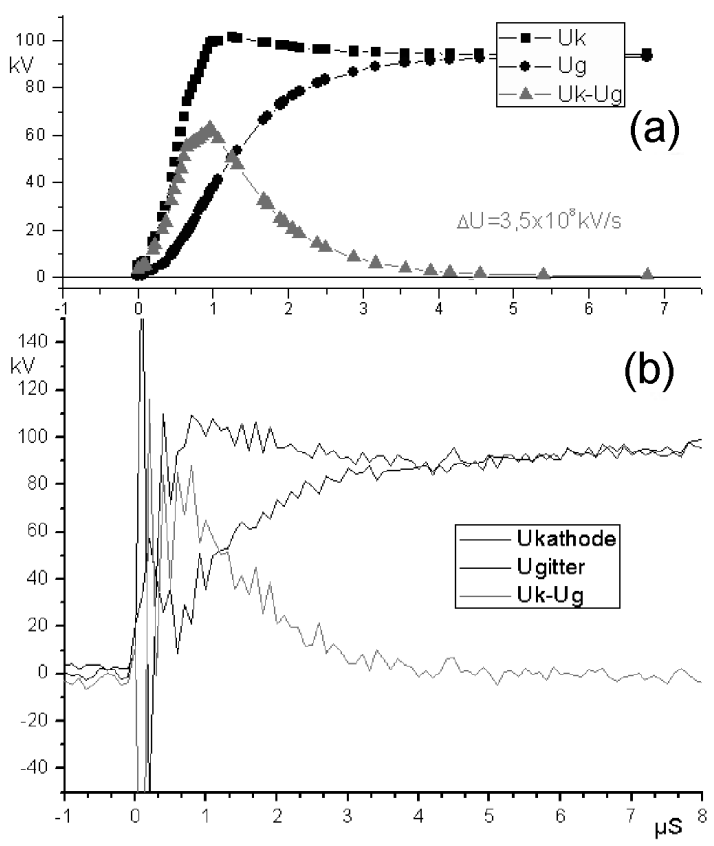

Fig. 5. Cathode $U_{\mathrm{k}}$ and grid voltage $U_{\mathrm{g}}$ and their difference: (a) simulation PsPice, (b) experimental values.

In addition to that a reduction in working pressure allows to delay or even prohibits the anode plasma formation. A clean surface of the target is required in any case.

\section{References}

[1] V. Engelko, B. Yatsenko, G. Müller, H. Bluhm, Vacuum 62, 211 (2001).

[2] G. Müller, V. Engelko, A. Weisenburger, A. Heinzel, Vacuum 77, 469 (2005).

[3] A. Weisenburger, E. Landhäußer, G. Müller, Ch. Puls, in: Proc. Int. Conf. on Gears VDI Berichte 2004, 2, München 2005, p. 1055.

[4] A. Weisenburger, G. Rizzi, A. Scrivani, Georg Mueller, J.R. Nicholls, Surf. Coatings Technol. 202, 704 (2007).

[5] A. Weisenburger, A. Heinzel, C. Fazio, G. Müller, V.G. Markov, A.D. Kastanov, J. Nucl. Mater. 377, 261 (2008).

[6] A. Weisenburger, A. Heinzel, G. Müller, H. Muscher, A. Roussanov, J. Nucl. Mater. 376, 274 (2008). 